\title{
Generalization of fixed-interval response patterning in pigeons*
}

\author{
THOMAS D. NELSON and G. WILLIAM FARTHING \\ University of Maine, Orono, Maine 04473
}

\begin{abstract}
Three pigeons were trained on a multiple schedule in which keypecking was reinforced on a fixed-interval (FI) schedule in the presence of a vertical line and on a variable-interval (VI) schedule in the presence of a horizontal line. Response rates in the former component were positively accelerated (the "FI scallop"), whereas response rates in the second component were relatively constant. In a subsequent generalization test on the line-tilt continuum, the index of curvature decreased systematically as the line was rotated from vertical to horizontal in 30 -deg steps. Frequency distributions of indices of curvature suggested that intermediate average index values for intermediate generalization test stimuli were mainly the result of intermediate response patterns in the individual test stimulus presentation, rather than the averaging of VI-like patterns during some stimulus presentations with FI-like patterns during other stimulus presentations.
\end{abstract}

In a typical stimulus generalization experiment (e.g., Guttman \& Kalish, 1956), a S is first trained to make a conditioned operant response in the presence of a particular stimulus, with responses reinforced on, usually, a variable-interval schedule. Then, during extinction, several different stimulus values along one stimulus dimension are presented in a random order, and the number of responses to each stimulus is determined. The typical generalization gradient, in which the number of responses decreases as the test stimulus becomes less and less similar to the training stimulus, is usually interpreted as representing a simple decrease in "response strength." Response strength is, of course, an abstract concept, and response rate is only one of several possible indices of response strength. But, whatever measured dependent variable is used, it is usually assumed that response strength itself is a continuous variable that can be measured in a continuous fashion, as in, for example, the case of generalization gradients showing decreasing average response rates as a function of changes in the test stimulus.

Ray and Sidman (1970) have summarized evidence that response rate may be a poor measure to use in studies of stimulus control. In fact. it may be misleading to assume that changes in responding as a function of stimuli can be described simply in terms of changes in response strength per se, where response strength is assumed to be a continuous variable. For example, Blough (1963) has shown that many of the interresponse times during a wavelength generalization test in pigeons have the same short value, regardless of the test stimulus wavelength. These short interresponse times are apparently controlled by internal stimuli, and only the longer interresponse times are controlled by the external

\footnotetext{
* This research was supported by Grant 18290 from the National Institute of Mental Health to G.IV.J. and by a NDLA Title IV fellowship awarded to T.D.N. Reprints inas be obtained from G. William larthing. Department of Psychology. 301 little Hall. L'njersity of Maine. Orono. Majne 04473.
}

stimulus value. Migler (1964), Migler and Millenson (1969), and Cumming and Eckerman (1965) did experiments in which a $S$ was trained to make two different responses (defined either temporally or spatially) in the presence of two different stimuli and then was tested with intermediate stimulus values. The results of these experiments showed that at intermediate stimulus values the response on individual trials was typically one or the other of the two responses that had previously been trained rather than some intermediate response. The conclusion to be drawn from these experiments is that the apparent intermediate response rate (or other measure of response strength) to a novel test stimulus in stimulus generalization experiments may not, in fact, represent a simple intermediate value of an underlying continuous variable, response strength. Rather, the apparent intermediate value of the dependent variable may represent the averaging of two or more different responses which the organism makes in the presence of the test stimulus, and the generalization gradient is a result of averaging different proportions of the different responses in the presence of the different stimulus values.

In view of the limitations of response rate as a dependent variable in stimulus generalization research, it is important to learn more about how other dependent variables are affected by novel stimuli in generalization experiments. One essential approach is to use discrete trial procedures, as advocated by Ray and Sidman (1970). However, in order to have a thorough understanding of stimulus control, it is also necessary to use dependent variables even more complex than simple response rate, such as the degree of curvature in a fixed-interval response pattern. which is a measure of rate change as a function of time. In studying the curvature of the fixed-interval response pattern, the $E$ does not assume that an average response rate to a stimulus is an adequate indication of the effect of the stimulus on the S's response. but. rather. he explicitl? 
examines the pattern of response rate change during test stimulus presentations.

Konick and Thomas (1968) trained pigeons to peck at a response key illuminated by $555-\mathrm{nm}$ light, with reinforcement on a fixed-interval 60-sec schedule. and then administered a wavelength generalization test in extinction. Typical generalization decrements in average response rate to the novel test stimuli occurred. However. the relative fixed-interval response patterns during the stimulus presentations were essentially the same for all of the test stimuli (except the 606-nm value. to which virtually no responding occurred). That is, response rates during the successive quarters of the 60 -sec test stimulus period were multiplicatively related so that. by way of illustration, if there were $4,6,8$. and 12 responses per quarter to one stimulus, there would be approximately 2.3.4. and 6 responses to a stimulus that produced a lower overall average response rate. In other words. there was apparently no systematic gradient of changes in the curvature of the fixed-interval response pattern as a function of test stimulus value.

The purpose of the present experiment was to further study generalization of the fixed-interval response pattern. The failure of Konick and Thomas (1968) to find a systematic generalization gradient of the fixed-interval response pattern apparently indicates that, in their procedure. the response pattern was not controlled by the external wavelength stimulus. Although the wavelength stimulus controlled the overall average response rate. the particular pattern of responding was apparently controlled only by the schedule of reinforcement. In the present study, the pattern of responding was brought under exteroceptive stimulus control by using a multiple schedule in which one stimulus value (vertical line) was associated with a fixed-interval (FI) schedule of reinforcement and a different stimulus value (horizontal line) was associated with a variable-interval (VI) schedule of reinforcement. This procedure produced a typical FI positively accelerated or "scalloped" response rate pattern in the presence of the vertical line and a relatively constant rate of response in the presence of the horizontal line. Finally, a generalization test on the line-tilt continuum was administered in order to examine changes in the response pattern as a function of changes in the exteroceptive stimulus value.

The present study examined generalization of the fixed-interval response pattern, as indicated by a mathematical index of curvature (Fry, Kelleher, \& Cook. 1960). In addition. this study attempted to determine whether a change in the average degree of curvature as a function of test stimuli is the result of the $S$ responding in an intermediate pattern during individual presentation of the intermediate test stimuli or, alternatively. the result of averaging two different response patterns, FI-like and VI-like. where one pattern occurs during some test stimulus presentations and the other occurs during other test stimulus presentations.

\section{METHOD}

\section{Subjects}

Three experimentally najve female Silver King pigeons (5-8 years old) were maintained at $80^{\circ} \pm 15 \mathrm{~g}$ of their free-feeding body weights.

\section{Apparatus}

The birds were trained in a Lehigh Valley Electronics (Model 1519) pigeon test chamber. which included a transparent response key that was mounted behind a 1 -in.-diam circular opening above the feeder opening. An in-line digital display cell was used to transilluminate the key with a white line (1 $\times 1 / 8$ in.) on a black background. The line could be presented at any of six angles of orientation: $0 \mathrm{deg}$ (vertical), $\pm 30 \mathrm{deg}$. $\pm 60 \mathrm{deg}$, and $90 \mathrm{deg}$ (horizontal). Reinforcers consisted of $3 \mathrm{sec}$ access to mixed grain. Extraneous noises were masked by a fan in the test chamber and a white noise in the room. Relay circuitry and recording equipment were located in an adjoining room.

\section{Procedure}

The birds were first trained to peck at the vertical line on the response key and given 30 continuous reinforcements (CRF) per day' for 3 days. The houselight was on during initial shaping but was faded out during the second CRF session and remained off for the duration of the experiment. Then the birds were given one session with reinforcement on a FI 40-sec schedule and five sessions on a FI 80-sec schedule. In these preliminary FI sessions. the first peck after 40 (or 80 ) sec produced a reinforcer. terminated the stimulus on the key, and started a 10-sec blackout. These $\mathrm{FI}$ sessions terminated after 60 reinforcements.

Following the preliminary training. all birds were given 25 sessions of training on a VR 3 (FI 80-sec) schedule. Only one-third of the stimulus periods terminated with a reinforcement: the other two-thirds of the stimulus periods terminated with the first peck after $80 \mathrm{sec}$. with no reinforcement. As before. stimulus periods were separated by 10 -sec blackouts. These sessions terminated after 20 reinforcements.

The final phase of training involved 80 sessions of discrimination training on a multiple VR 3 (FI 80-sec) vs VI $240-\mathrm{sec}$ schedule. The VR 3 (FI 80-sec) (vertical line) component alternated randomly with an equal number of $\mathrm{VI}$ $240-\mathrm{sec}$ (horizontal line) components. All FI components terminated with the first peck after $80 \mathrm{sec}$. Whereas VI components terminated automatically after $80 \mathrm{sec}$; all stimulus periods were separated by $10-\mathrm{sec}$ blackouts. In each component a reinforcement could occur once every $240 \mathrm{sec}$ on the average. In the FI component. reinforcement could be produced by the furst peck at the end of an 80-sec stimulus period (for one-third of the periods). while reinforcement might be produced at any time during an $80-\mathrm{sec}$ stimulus period in the VI component. These multiple-schedule training sessions terminated after 20 reinforcements.

Finally, all birds were given a warm-up of additional multiple-schedule training until 10 reinforcers had been collected and then a generalization test in extinction. Each of the six line-tilt stimuli was presented once in each of 12 randomized blocks. During the test. the $E$ recorded the number of keypeck responses during each 20-sec quarter of each $80-\mathrm{sec}$ stimulus period. Test stimulus periods were separated b! 10-sec blackouts.

\section{RESULTS}

The degree of response rate acceleration within the 
80-sec stimulus periods was analyzed in terms of an index of curvature (Fry, Kelleher, \& Cook, 1960): For an interval divided into quarters, the maximum and minimum values of the index are +0.75 and -0.75 , indicating all responses occur in the fourth quarter and all responses occur in the first quarter, respectively; the index is 0.00 when there are an equal number of responses in each of the four quarters.

The average indices of curvature for Birds 1,2, and 3 for the last two sessions of preliminary FI 80-sec training were $0.15,0.27$, and 0.25 , respectively. For the last five sessions of VR 3 (FI 80-sec) training, the indices were $0.20,0.14$, and 0.13 , respectively. For the last 10 sessions of mult VR 3 (FI 80-sec) vs VI 240-sec training, the average indices in the FI component (and the VI
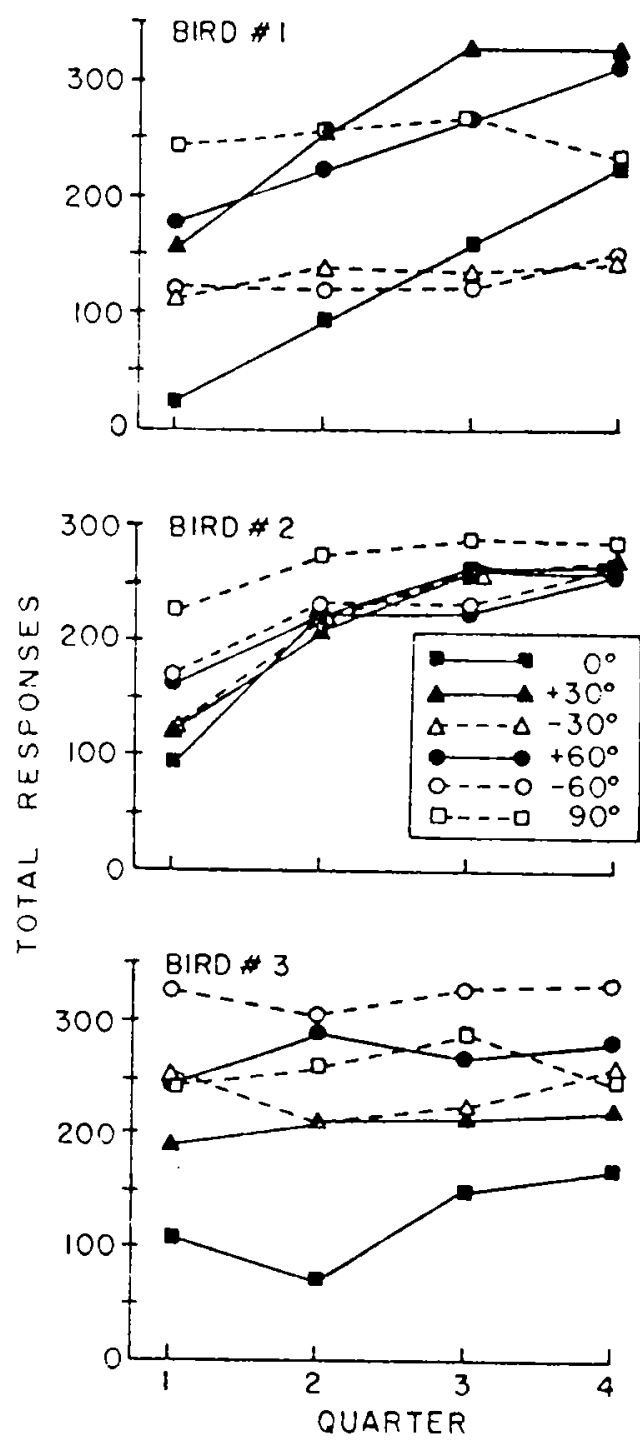

Fig. 1. Total number of responses to each generalization test stimulus during each $20-\mathrm{sec}$ quarter of the $80-\mathrm{sec}$ stimulus presentations for individual Ss. During training. keypecking had been reinforced on a VR 3 (FI 80-sec) schedule in the presence of the 0 -deg line and on a VI 240-sec schedule in the presence of the 90-deg line.

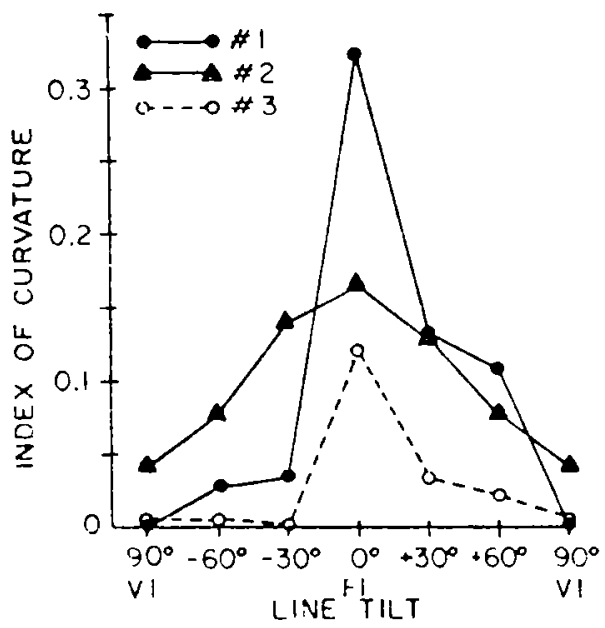

Fig. 2. Average indices of curvature for each of the generalization test stimuli for individual Ss. These average indices are based on the total response data shown in Fig. 1.

component, in parentheses) were $0.21(0.04), 0.19$ $(0.04)$, and $0.12(0.00)$, respectively. For the three birds, the average response rate increased from $31.2 / \mathrm{min}$ in the first quarter to $83.1 / \mathrm{min}$ in the fourth quarter of the $\mathrm{FI}$ component but only from 63.2 to $71.9 / \mathrm{min}$ in the VI component during the last 10 training sessions.

Examination of individual cumulative records obtained during the mult VR 3 (FI 80-sec) vs VI 240-sec training indicated that the FI "scalloped" response pattern in the presence of the vertical line was typically a period of no responding when the vertical line was presented, followed by an abrupt shift to a high rate of responding until the end of the $80-\mathrm{sec}$ period (break and run pattern). The length of the pause in the FI component was variable, but the pause occurred reliably. In the presence of the horizontal line, a break and run pattern was rarely observed and a steady response rate was typical. In general, responding was more variable in the presence of the vertical (FI) line than the horizontal (VI) line.

Figure 1 shows the total number of responses to each generalization test stimulus during each 20 -sec quarter of the 80-sec stimulus presentations. Birds 1 and 2 had about equal average response rates in the fourth quarter of the 0-deg (FI) and 90-deg (VI) line stimuli during the test, but Bird 3 responded considerably less to the 0-deg line than to the 90-deg line, even in the fourth quarter.

Figure 2 shows the overall average index of curvature for the pattern of responding during each test stimulus for individual Ss. This average index of curvature was calculated from the response totals shown in Fig. 1. The average index of curvature was highest at the 0 -deg (FI) stimulus for each bird and decreased svstematically as the line tilt approached the $90-\operatorname{deg}$ (VI) stimulus.

In order to analyze the generalization test data in more detail. indices of curvature were calculated for each individual 80-sec test stimulus presentation. (A small number of test stimulus periods where no responses aceured were omitted form the further 


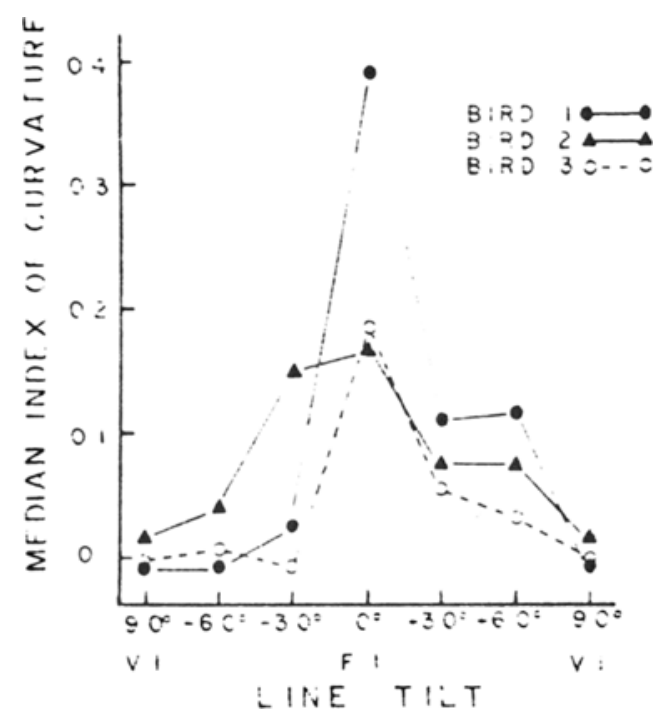

Fig. 3. Median indices of curvature for each of the generalization test stimuli for individual Ss. These medians were calculated from the indices of curvature for each of the individual stimulus presentations during the generalization test.

analysis presented in Figs. 3 and 4: There were nine test stimulus presentations in which no responding occurred for Bird 1. one for Bird 2, and two for Bird 3 out of 72 total test stimulus presentations for each bird.) Figure 3 shows the medians of the indices of curvature for the individual test stimulus presentations for each of the test stimuli for each $\mathrm{S}$. The median index of curvature was highest for the 0-deg (FI) stimulus. and the median decreased more or less systematically as the line tilt approached the 90-deg (VI) stimulus.

In order to determine whether the average indices shown in Fig. 2 could be attributed to the averaging together of FI-like and VI-like patterns of responding during the different individual test stimulus presentations. it was necessary to categorize the index for each individual 80-sec test stimulus period as being a typical fixed-interval index value, a typical variable-interval index value. or some intermediate value. Such a decision must be somewhat arbitrary, since the index of curvature is a continuous measure and, even during reinforced training, there is some variability in the value of this index on both fixed- and variable-interval schedules.

For purposes of analyzing the generalization test data. four categories of index of curvature were established. The specific range of index values in each category was defined independently for each S. depending on each S's own behavior during the generalization test. Category 1 was defined as including all indices of curvature at or below the S's median index for the 90-deg line (VI component). Category 4 was defined as including all indices at or above the S's median index for the 0-deg line (FI component). (The median indices for 0 and 90 deg are shown in Fig. 3.) Categories 2 and 3 were defined by bisecting the interval between Categories 1 and 4.
Figure 4 presents frequency distributions of the indices of curvature for individual test stimulus presentations sorted into the four index categories for each test stimulus for each bird. The left column of graphs shows the frequency distributions for the 0 . and 90-deg training stimuli. For each bird the frequency distributions for the 0 - and 90-deg stimuli are clearly different. though there is some overlap between the distributions. Frequency distributions for the -30 - and -60-deg test stimuli are shown in the middle column. whereas distributions for the +30 - and +60 -deg stimuli are shown in the right column. The meaning of the frequency distributions will be discussed below.

All three birds responded in all 12 blocks of stimuli during the generalization test. In general, there were no systematic changes in the indices of curvature during the course of testing in extinction.

\section{DISCUSSION}

In the present experiment, the mean (and median) index of curvature changed sy'stematically as a function of generalization test stimulus value (see Figs. 2 and 3 ). Contrasted with Konick and Thomas's (1968) experiment. in which this did not occur, the present results suggest that training on a multiple schedule involving two stimuli, associated with different schedules of reinforcement that generate different typical response patterns, is a sufficient condition for producing exteroceptive stimulus control of response pattern and
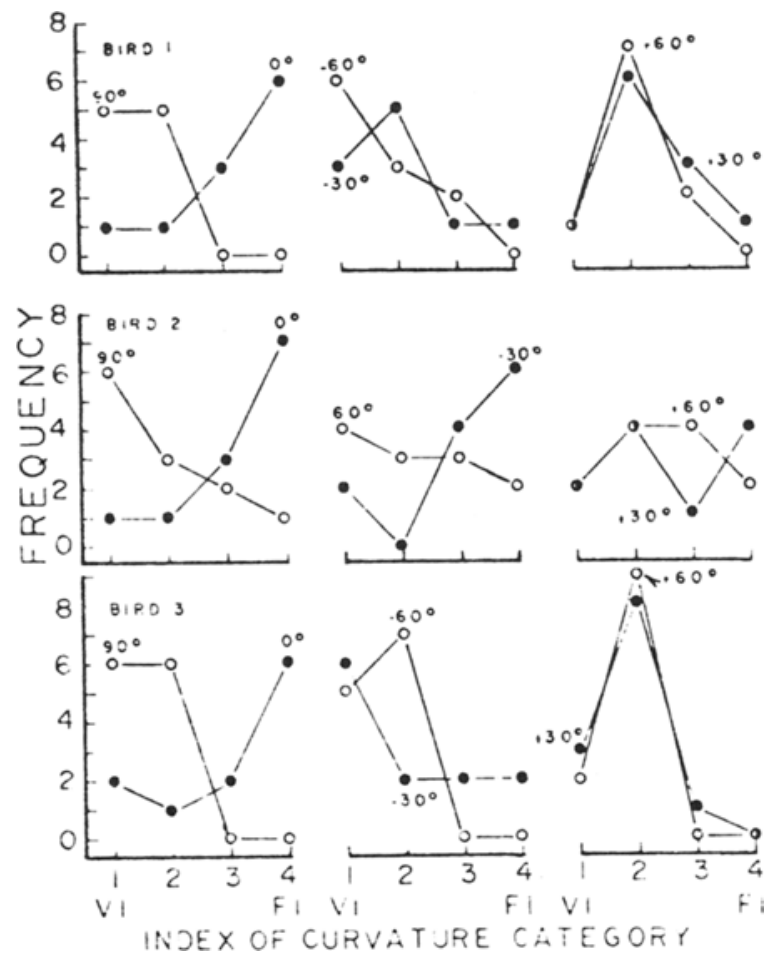

Fig. 4. Frequency distributions of indices of curvature for each of the generalization test stimuli for individual Ss. See text for an explanation of Index Categories 1. 2. 3. and 4. 
systematic generalization gradients of response pattern

Of particular interest in the present experiment is the question of whether the intermediate average index of curvature produced by some of the intermediate test stimulus values is a result of an actual intermediate degree of curvature, typically occurring during individual test stimulus periods, or a result of the averaging of relatively low (VI) indices occurring in some test periods with relatively high $(\mathrm{FI})$ indices occurring in other test periods.

The index of curvature frequency distribution data in Fig. 4 are best interpreted while referring back to the mean index values presented in Fig. 2. It would be expected that, if intermediate average indices to intermediate stimulus values were simply the result of ave raging low (VI) and high (FI) indices, then in these cases the frequency distributions should be bimodal, with most of the indices falling into Categories 1 and 4 . On the other hand, if intermediate average response indices represent intermediate typical response patterns, then the frequency distributions should show relatively more indices in Categories 2 and/or 3 than in Categories 1 and 4.

Most of the correlations between the mean index of curvature in Fig. 2 and the shape of the index frequency distribution for that stimulus in Fig. 4 support the conclusion that mean index values that are intermediate between the 0 - and 90-deg means are mainly a result of intermediate typical index values for individual test stimulus presentations rather than the averaging of low (VI-like) indices for some stimulus presentations with high (FI-like) indices for other stimulus presentations.

In Fig. 2 there are 10 cases in which the mean index for a test stimulus is intermediate between the mean indices of the 0 - and $90-\mathrm{deg}$ stimulus for that bird. (For Bird 3 the mean indices for the -30 - and -60 -deg stimuli are approximately equal to the mean index for the 90-deg stimuli.) The index frequency distributions in Fig. 4 for these 10 cases fall into three groups: (1) In six cases, most of the indices fall into intermediate Categories 2 and/or 3 . The most striking cases are the +30 - and +60-deg stimuli in Birds 1 and 3, where there is a clearly defined mode in Index Category 2. For Bird 1 the mode for the -30 -deg stimulus is in Category 2. For Bird 2 the +60-deg stimulus has more indices in both Categories 2 and 3 than in either Category 1 or 4 . (2) In two cases, the mode of the frequency distribution is clearly in one of the extreme categories. For Bird 1, the mode of the -.60-deg distribution is clearly in Category 1 and, in fact, the mean for $-60 \mathrm{deg}$ is not much greater than the mean for $90 \mathrm{deg}$. For Bird 2. the mode for the -30-deg distribution is clearly in Category 4 , and the mean for - 30 deg is almost as great as the mean for $0 \mathrm{deg}$. (3) In two cases, the frequency distributions cannot be clearly classitied into either Group 1 or 2 above. For Bird 2. the -60-deg stimulus has a slight mode in Catcgory 1 but also clearly more indices in Categuries 3 and 4 . compared to the 40 -d dis stimulus. Also, for Bird 2, the +30.deg stimulus has modes in Categories 2 and 4 that make it difficult to classify. None of the 10 cases of intermediate mean indices have frequency distributions that clearly support the notion that irtermediate mean values result from averaging mostly high and low values, al though perhaps the two cases in Group 3 come closest to supporting this possibility. Of the two mean indices in Bird 3 that were not intermediate between 0 - and 90-deg means but were about equal to the 90-deg mean, the -30-deg frequency distribution has a clear mode in Category 1 , whereas the -60-deg distribution differs from the 90-deg distribution by only one index apparently shifted from Category 1 to Category 2.

In general, the correlations between the shapes of the frequency distributions (Fig. 4) and the median indices (Fig. 3) are better than the correlations between the frequency distributions and the mean indices (Fig. 2). This is not surprising, since the index categories used in Fig. 4 were based on the median indices of the 0 - and 90-deg test stimuli.

In conclusion, this study demonstrates that systematic generalization gradients of an index of curvature can be obtained following training on a multiple FI VI schedule. The results further suggest that intermediate average indices of curvature obtained with intermediate test stimuli are mainly the result of intermediate response patterns in individual presentations of the intermediate stimuli, rather than simply the averaging of VI-like patterns in some stimulus periods with FI-like patterns in other stimulus periods. The present study leaves unanswered the question of whether the Ss might alternate between VI-like and FI-like behavior during the course of single stimulus presentations, which would result in an intermediate index of curvature for the individual stimulus presentation period. An answer to this question will require a finer-grained analysis and should probably involve a maintained generalization gradient procedure, rather than testing in extinction, in order to reduce the variability in average response rates in the individual test stimuli and obtain more reliable response patterns.

\section{REFERENCES}

Blough. D. S. Interresponse time as a function of continuous variables: A new method and some data. Journal of the Experimental Analysis of Behavior. 1963.6. 237-246.

Cumming. W. W.. \& Eckerman. D. A. Stimulus control of a differentiated operant. Psychonomic Science. 1965. 3. 313-314

Fry. W.. Kelleher. R. T.. \& Cook. L. A mathematical index of performance on fixed-interval schedules of reinforcement. Journal of the L xperimental Analysis of Bchavior. 1960. 3. 193.199.

Guttman. N. K Kalish. H. I. Discriminability and stimulus generalization. Journal of Experimental Psichology. 1956.51. 79-88.

honick. D. S. \& [homis. D. R. Simulus generalization following fied intersal training. Iournal of leprintental puchulug: 1968. 77. $689-691$. 
Migler. B. Effects of averaging data during stimulus generalization. Journal of the Experimental Analysis of Behavior. 1964. 7. 303-307.

Migler, B.. \& Millenson. J. R. Analysis of response rates during stimulus generalization. Journal of the Experimental Analysis of Behavior. 1969. 12.81-87.

Ray. B. A.. \& Sidman. II. Reinforcement schedules and stimulus control. In W. If. Schoenfield (Fd.). The theory of reinforcement schedules. IeW York: Appleton-Century-Crofts. 1970. Pp. 187-214.

(Received for publication July 13. 1972: revision received Warch 29. 1973.) 